\title{
Discretized Lyapunov Function Approach for Switched Linear Systems under Dwell Time Constraint
}

\author{
Yongchi Zhao, ${ }^{1,2}$ Shengxian Zhuang, ${ }^{1}$ Weiming Xiang, ${ }^{3}$ and Lin $\mathrm{Du}^{3}$ \\ ${ }^{1}$ School of Electrical Engineering, Southwest Jiaotong University, Chengdu 610031, China \\ ${ }^{2}$ Institute of Information Security and Computing Technology, Mianyang Normal University, Mianyang 621006, China \\ ${ }^{3}$ School of Transportation and Logistics, Southwest Jiaotong University, Chengdu 610031, China \\ Correspondence should be addressed to Yongchi Zhao; dong0824@sohu.com
}

Received 14 March 2014; Accepted 18 June 2014; Published 3 July 2014

Academic Editor: Jaeyoung Chung

Copyright (C) 2014 Yongchi Zhao et al. This is an open access article distributed under the Creative Commons Attribution License, which permits unrestricted use, distribution, and reproduction in any medium, provided the original work is properly cited.

\begin{abstract}
This paper is concerned with the stability and disturbance attenuation properties of switched linear system with dwell time constraint. A novel time-scheduled Lyapunov function is introduced to deal with the problems studied in this paper. To numerically check the existence of such time-scheduled Lyapunov function, the discretized Lyapunov function technique usually used in timedelay system is developed in the context of switched system in continuous-time cases. Based on discretized Lyapunov function, sufficient conditions ensuring dwell-time constrained switched system global uniformly asymptotically stable are established, then the disturbance attenuation properties in the sense of $\mathscr{L}_{2}$ gain are studied. The main advantage of discretized Lyapunov function approach is that the derived sufficient conditions are convex in subsystem matrices, which makes the analysis results easily used and generalized. Thus, the $\mathscr{H}_{\infty}$ control synthesis problem is considered. On the basis of analysis results in hand, the control synthesis procedures including both controller and switching law design are unified into one-step method which explicitly facilitates the control synthesis process. Several numerical examples are provided to illustrate the results within our paper.
\end{abstract}

\section{Introduction and Some Preliminaries}

The switched systems have emerged as an important class and represent a relatively new and very active area of current research in the field of control systems [1-4]. A switched system is composed of a family of continuous or discretetime subsystems, described by differential or difference equations, respectively, along with a switching rule governing the switching between the subsystems. The motivation for studying such switched systems comes from the fact that switched system can be efficiently used to model many practical systems that are inherently multimodel in the sense that several dynamical subsystem models are required to describe their behavior, such as several real-world, and industrial processes exhibit switched and hybrid nature intrinsically [5$8]$ and from the fact that switching control strategies also arise in many engineering applications to improve control performance [9-11].
In this paper, we consider the switched linear system described by

$$
\begin{aligned}
& \dot{x}(t)=A_{\sigma(t)} x(t)+B_{\sigma(t)} \omega(t), \\
& y(t)=C_{\sigma(t)} x(t)+D_{\sigma(t)} \omega(t),
\end{aligned}
$$

where $x(t) \in \mathbb{R}^{n}$ is the system state and $\omega(t) \in \mathbb{R}^{m}$ is the energy-bounded disturbance input. $\sigma(t)$ is a piecewise constant function of time, called switching law or switching signal, which takes value in a finite index set $\mathscr{I}:=\{1,2, \ldots, N\}$. $N>0$ is the number of subsystems. System matrices $A_{i}, B_{i}$, $C_{i}$, and $D_{i}$ are constant with appropriate dimensions. Let the discontinuity points of $\sigma(t)$ be denoted by $t_{k}$, and let $t_{0}$ stand for the initial time by convention; the switching sequence can be described as $\mathcal{S}:=\left\{t_{0}, t_{1}, t_{2}, \ldots, t_{k}, \ldots\right\}$. Calling $\mathscr{D}_{\tau}$ the set of all switching policies with dwell time $\tau$, that is the set of all $\sigma(t)$ for which the time interval between successive discontinuities of $\sigma(t)$ satisfies $t_{k+1}-t_{k} \geq \tau, k=0,1,2, \ldots$. 
Generally, the stability and disturbance attenuation properties are the main concerns in the field of switched system such as [3, 4, 12-23], which are also the main issues considered in this paper. At first, we introduce some relevant conceptions, problems which we concentrate on, and review of several existing results on stability and disturbance attenuation properties.

Definition 1. The equilibrium $x=0$ of system (1) with $\omega(t)=$ 0 is globally uniformly asymptotically stable (GUAS) under certain switching signal $\sigma(t)$ if, for initial condition $x\left(t_{0}\right)$, there exist a class $\mathscr{K} \mathscr{L}$ function $\beta$ such that the solution of the system satisfies $\|x(t)\| \leq \beta\left(\left\|x\left(t_{0}\right)\right\|, t\right), \forall t \geq t_{0}$. (A function $\gamma:[0,+\infty) \rightarrow[0,+\infty)$ is a $\mathscr{K}$ function if it is strictly increasing and $\gamma(0)=0$, and also a function $\beta$ : $[0,+\infty) \times[0,+\infty) \rightarrow[0,+\infty)$ is a $\mathscr{K} \mathscr{L}$ function if for each fixed $s$ the function $\beta(r, s)$ is a $\mathscr{K}$ function with respect to $r$, and for each fixed $r$ the function $\beta(r, s)$ is decreasing with respect to $s$ and $\beta(r, s) \rightarrow 0$ as $s \rightarrow 0$.)

One of the basic problems for switched system (1) with dwell-time constrained switching signal is to identify or determine the minimal value of dwell time $\tau$ and the set of switching law set $\mathscr{D}_{\tau}$ such that system (1) with $\omega(t)=0$ is GUAS, that is, the computation on minimal admissible dwell time $\tau$ guaranteeing GUAS of system (1).

Problem 2. Given switched system (1), find the minimal value of dwell time $\tau^{*}$ such that switched system (1) is GUAS for any $\tau \geq \tau^{*}$.

A famous result for above stability analysis problem is given incorporated with multiple Lyapunov function (MLF) formulated as $V_{i}(t)=x^{T}(t) P_{i} x(t), i \in \mathscr{I}$, where $P_{i}>0, \forall i \in$ $\mathscr{I}$, which is an efficient stability analysis tool for switched systems. Based on MLF, the following lemma provides sufficient conditions ensuring system (1) GUAS for continuoustime case [24-26].

Lemma 3. Consider the switched linear system (1) with $\omega(t)=$ 0 and let $\lambda>0, \mu>1$ be given constants. If there exist a set of positive definite symmetric matrices $P_{i}, i \in \mathscr{I}$, such that $A_{i}^{T} P_{i}+$ $P_{i} A_{i}<-\lambda P_{i}, \forall i \in \mathscr{I}$, and $P_{i} \leq \mu P_{j}, i \neq j, \forall i, j \in \mathscr{I}$, then, system (1) is GUAS for any switching law $\sigma(t) \in \mathscr{D}_{\tau}$, where dwell time satisfies $\tau>\tau^{*}=\ln \mu / \lambda$.

Lemma 3 provides us a method to compute the admissible dwell time of switched linear system (1); however, the parameters $\mu$ and $\lambda$ often need to be chosen manually in advance, and moreover, as the main disadvantage of Lemma 3, the estimation of admissible dwell time by $\tau>\tau^{*}=\ln \mu / \lambda$ is nonconvex in nature; thus, the obtained minimal admissible dwell time $\tau$ is usually significantly conservative by some inappropriate choices of parameters $\mu$ and $\lambda$ in the framework of MLF approach. Hereby, to improve Lemma 3, we have to establish new sufficient conditions that are in convex form essentially.

Then, another basic problem of switched system (1) with dwell-time constrained switching signal is how the $\mathscr{L}_{2}$ norm of $y(t)$ changes for any $\omega(t) \in \mathscr{L}_{2}[0, \infty)$. Here the $\mathscr{L}_{2}$ norm is defined as $\|v(t)\|_{\mathscr{L}_{2}}=\sqrt{\int_{0}^{\infty} v^{T}(t) v(t) \mathrm{d} t}$. The $\mathscr{L}_{2}$ gain performance for switched system (1) is defined as follows.

Definition 4. For a scalar $\gamma>0$, system (1) is said to be GUAS and has an $\mathscr{L}_{2}$ gain $\gamma$ under certain switching signal $\sigma(t)$ if, under zero initial condition, system (1) is GUAS when $\omega(t)=$ 0 , and the inequality $\|y(t)\|_{\mathscr{L}_{2}} \leq \gamma\|\omega(t)\|_{\mathscr{L}_{2}}$ holds.

Our objective is to characterize the relationship between $\mathscr{L}_{2}$ gain and dwell time. Particularly, the minimal value of $\gamma$ ensures the $\mathscr{L}_{2}$ gain inequality satisfied is called the $\mathscr{L}_{2}$ induced gain $\gamma^{*}$. The problem of computation has to be pointed out on the $\mathscr{L}_{2}$ induced gain versus dwell time which has been viewed as an open problem [27] and not completely solved so far.

Problem 5. Given switched system (1) with a dwell time $\tau$, determine the $\mathscr{L}_{2}$ induced gain $\gamma^{*}$ of switched system (1).

A natural solution for above problem is to extend the stability results in Lemma 3 to $\mathscr{L}_{2}$ gain analysis problem by some standard techniques. Unfortunately, Lemma 3 cannot be extended straightforwardly to solve the open problem on the $\mathscr{L}_{2}$ gain performance with dwell time. Under the framework of MLF, it has been proved that the straightforward extension of Lemma 3 only guarantees the undesirable weighted $\mathscr{L}_{2}$ gain [28-30] described as $\int_{0}^{\infty} e^{-\lambda t} y^{T}(t) y(t) \mathrm{d} t \leq$ $\gamma^{2} \int_{0}^{\infty} \omega^{T}(t) \omega(t) \mathrm{d} t$, where $\lambda>0$. Obviously the weighted $\mathscr{L}_{2}$ gain is not an anticipated performance in both mathematical analysis and practical use. The constant (nonweighted) $\mathscr{L}_{2}$ gain analysis problem still remains unsolved, though some other important advances for constant $\mathscr{L}_{2}$ gain performance have been reached recently. In $[19,20]$, the authors showed that $\mathscr{L}_{2}$ gain stability can be characterized by the existence of a convex homogeneous (of degree two) Lyapunov function, though the construction of such a storage function (by solving the Hamilton-Jacobi inequalities) still remains a theoretical challenge. And in [31-33], the corresponding conditions are nonconvex in system matrices, such as terms $e^{\left(A_{i}+B_{i} L_{i}\right) \tau},\left(A_{i}+B_{i} L_{i}\right)^{\tau}$, or $A_{i}^{\tau}, A_{i}^{\tau-1}, A_{i}^{\tau-2}, \ldots$, which are involved in linear matrix inequities (LMIs) conditions, which significantly restricts their further application such as $\mathscr{H}_{\infty}$ controller synthesis problem.

Therefore, the key point but remaining open is to find convex sufficient conditions to establish switched system (1) GUAS associated with dwell time and generalize to $\mathscr{L}_{2}$ gain performance to solve the open problem in [27], which is also our main objective in this paper.

The basic structure of our main results for switched system (1) is as follows. At the first step we propose a novel time-scheduled Lyapunov function and provide a computable method, called discretized Lyapunov function technique, to determine the time-scheduled Lyapunov function in continuous-time case. The second step involves applying discretized Lyapunov function approach into stability analysis and $\mathscr{L}_{2}$ gain performance issues for switched system (1). The main feature of our approach is that the derived sufficient conditions are affine in system matrices, which 
meets the main objective in our paper. Finally, with our analysis results in hand, we turn to control synthesis and derive explicit controller formulas which ensure stability and $\mathscr{H}_{\infty}$ performance the switched system in closed loop. In the context of control synthesis, it naturally contains feedback controller and switching law design. Unlike most of previous results in which controller and switching law design are split in two separate steps, a one-step design methodology is presented on the basis of discretized Lyapunov function approach; as far as we know, this is possible to be the first result that unifies the controller and switching law into one step in the synthesis process. Along with this paper, several academic examples are provided to illustrate the advantages of our approach.

Some Notations. The superscript $T$ stands for matrix transposition, $\mathbb{R}^{n}$ denotes the $n$ dimensional Euclidean space, $\mathbb{R}^{+}$ stands for the set of nonnegative numbers, and $\mathbb{Z}^{+}$represents the set of nonnegative integers. The notations $\|\cdot\|$ and $\|\cdot\|_{\mathscr{L}_{2}}$ refer to the Euclidean norm and $\mathscr{L}_{2}$ norm, respectively. In addition, in symmetric block matrices, we use $*$ as an ellipsis for the terms that are introduced by symmetry and diag $\{\cdots\}$ stands for a block-diagonal matrix. The notation $P>0$ $(P \geq 0)$ means $P$ is real symmetric and positive definite (semipositive definite). $\operatorname{He}\{A\}$ is the shorthand notation for $A^{T}+A$. I stands for identity matrix.

\section{Discretized Lyapunov Function}

The main idea within this paper is to construct a new type of time-scheduled Lyapunov function in the form of

$$
V_{i}(t)=x^{T}(t) \mathscr{P}_{i}(t) x(t), \quad i \in \mathscr{F},
$$

where $\mathscr{P}_{i}(t), i \in \mathscr{I}$, is a time-scheduled positive definite matrix. Explicitly, the time-scheduled Lyapunov function with matrix function $\mathscr{P}_{i}(t), i \in \mathscr{I}$, can provide more choices to construct Lyapunov function and yield less conservative results than MLF, since MLF is only a particular case of $\mathscr{P}_{i}(t)=P_{i}, i \in \mathscr{I}$. However, in practice, to numerically check the existence of such matrix function $\mathscr{P}_{i}(t), i \in \mathscr{I}$, especially in continuous-time case, is not an easy task. Thus, in this paper we resort to discretized Lyapunov function technique which has been widely used in time-delay system analysis problems, for example [34, Section 5.7]. The basic idea of discretized Lyapunov function technique is to divide the domain of definition of matrix function $\mathscr{P}_{i}(t), i \in \mathscr{I}$, into finite discrete points or smaller regions, thus reducing the choice of time-scheduled Lyapunov function into choosing a finite number of parameters.

In order to establish a computable method to determine the continuous time-scheduled Lyapunov function $\mathscr{P}_{i}(t), i \in$ $\mathscr{I}$, we attempt to divide the dwell time interval $\left[t_{k}, t_{k}+\tau\right)$ into a sequence of small segments and employ the discretized Lyapunov function technique commonly used in time-delay system [34, Section 5.7]. The discretized Lyapunov function technique for switched system (1) is given in detail as follows.

We divide the dwell-time interval $\left[t_{k}, t_{k}+\tau\right)$ into $L$ segments described as $\mathcal{N}_{k, q}=\left[t_{k}+\theta_{q}, t_{k}+\theta_{q+1}\right)$, $q=0,1, \ldots, L-1$ of equal length $h=\tau / L$, and then $\theta_{q}=$ $q h=q \tau / L, q=0,1, \ldots, L$. The continuous matrix function $\mathscr{P}_{i}(t), t \in\left[t_{k}, t_{k}+\tau\right)$ are chosen to be linear within each segments $\mathcal{N}_{k, q}, q=0,1, \ldots, L-1$. Letting $P_{i, q}=P_{i}\left(t_{k}+\theta_{q}\right)$, then since the matrix function $\mathscr{P}_{i}(t)$ is piecewise linear in dwell-time interval $\left[t_{k}, t_{k}+\tau\right)$; it can be expressed in terms of the values at dividing points using a linear interpolation formula, that is, for $0 \leq \alpha \leq 1, q=0,1, \ldots, L-1$

$$
\begin{aligned}
\mathscr{P}_{i}(t) & =\mathscr{P}_{i}\left(t_{k}+\theta_{q}+\alpha h\right) \\
& =\mathscr{P}_{i}^{(q)}(\alpha)=(1-\alpha) P_{i, q}+\alpha P_{i, q+1}, \quad t \in \mathcal{N}_{k, q},
\end{aligned}
$$

where $\alpha=\left(t-t_{k}-\theta_{q}\right) / h$. Then the continuous matrix function $\mathscr{P}_{i}(t)$ is completely determined by $P_{i, q}, q=0,1, \ldots, L$, in the dwell-time interval $\left[t_{k}, t_{k}+\tau\right)$. Afterwards, in the interval $\left[t_{k}+\right.$ $\left.\tau, t_{k+1}\right), \mathscr{P}_{i}(t)$ is fixed as a constant matrix $\mathscr{P}_{i}(t)=P_{i, L}$. Hence the discretized matrix function $\mathscr{P}_{i}(t), i \in \mathscr{I}$, for continuoustime case is described as

$$
\begin{aligned}
& \mathscr{P}_{i}(t) \\
& =\left\{\begin{array}{ll}
P_{i}^{(q)}(\alpha) & t \in \mathcal{N}_{k, q}, q=0,1, \ldots, L-1 \\
P_{i, L} & t \in\left[t_{k}+\tau, t_{k+1}\right),
\end{array} i \in \mathcal{F}\right.
\end{aligned}
$$

and the corresponding discretized Lyapunov function is

$V_{i}(t)$

$=\left\{\begin{array}{ll}x^{T}(t) \mathscr{P}_{i}^{(q)}(\alpha) x(t) & t \in \mathscr{N}_{k, q}, q=0,1, \ldots, L-1 \\ x^{T}(t) P_{i, L} x(t) & t \in\left[t_{k}+\tau, t_{k+1}\right),\end{array} \quad i \in \mathscr{I}\right.$.

Obviously, the number of division segments has to be $L \geq 1$ when the discretized Lyapunov function approach is used. If it is enforced that $L=0$, by (5), the discretized Lyapunov function $V_{i}(t), i \in \mathscr{I}$, is reduced to MLF form. Thus, the discretized Lyapunov function is also a generalized form of MLF in continuous-time case.

The discretized Lyapunov function technique provides us a computable method to determine the time-scheduled Lyapunov function in continuous-time case, and what is more important, in contrast to the MLF $V_{i}(t)=x^{T}(t) P_{i} x(t), i \in \mathscr{I}$, in which the matrices $P_{i}, i \in \mathscr{I}$, are completely independent of dwell time $\tau$, the discretized Lyapunov function is constructed with the aid of information of dwell time $\tau$, and this unique feature can make the resulting sufficient conditions for GUAS and $\mathscr{L}_{2}$ gain affine in system matrices, which is a convenient tool to deal with the stability and disturbance attenuation problems and will be shown in next sections.

\section{Stability Analysis}

In this section, our analysis results employ a family of discretized Lyapunov functions in the form of (5) for each subsystem. Motivated by the idea that the value of Lyapunov function is nonincreasing at switching instant [17], the following theorem can be obtained with the help of a family of discretized Lyapunov function in the form of (5). 
Theorem 6. Consider the switched linear system (1) with $\omega(t)=0$. If there exist a set of matrices $P_{i, q}>0, q=0,1, \ldots, L$, $i \in \mathscr{I}$, such that

$$
A_{i}^{T} P_{i, q}+P_{i, q} A_{i}+\Psi_{i}^{(q)}<0, \quad q=0,1, \ldots, L-1, \forall i \in \mathscr{I}
$$

$$
A_{i}^{T} P_{i, q+1}+P_{i, q+1} A_{i}+\Psi_{i}^{(q)}<0, \quad q=0,1, \ldots, L-1, \forall i \in \mathscr{I}
$$

$$
\begin{gathered}
A_{i}^{T} P_{i, L}+P_{i, L} A_{i}<0, \quad \forall i \in \mathscr{I} \\
P_{j, 0}-P_{i, L} \leq 0, \quad i \neq j, \quad \forall i, j \in \mathscr{I},
\end{gathered}
$$

where $\Psi_{i}^{(q)}=L\left(P_{i, q+1}-P_{i, q}\right) / \tau$, then system (1) is GUAS under switching signal with dwell time $\tau$.

Proof. Choosing the discretized Lyapunov function for $i$ th subsystem $V_{i}(t)=x^{T}(t) \mathscr{P}_{i}(t) x(t), i \in \mathscr{I}$, where matrix function $\mathscr{P}_{i}(t), i \in \mathscr{I}$, is discretized according to (5). Thus, we see $\dot{V}_{i}(t)=x^{T}(t) \dot{\mathscr{P}}_{i}(t) x(t)+2 \dot{x}^{T}(t) \mathscr{P}_{i}(t) x(t)$. In each discretized segment $\mathcal{N}_{k, q}$, the following equation can be derived:

$$
\begin{aligned}
\dot{\mathscr{P}}_{i}(t) & =\dot{\mathscr{P}}_{i}\left(t_{k}+\theta_{q}+\alpha h\right) \\
& =\dot{\mathscr{P}}_{i}^{(q)}(\alpha)=\left(P_{i, q+1}-P_{i, q}\right) \dot{\alpha}, \quad t \in \mathcal{N}_{k, q} .
\end{aligned}
$$

Due to $\alpha=\left(t-t_{k}-\theta_{q}\right) / h$, we have $\dot{\alpha}=1 / h$, where $h=\tau / L$. Hence $\dot{\mathscr{P}}_{i}(t)$ becomes

$$
\dot{\mathscr{P}}_{i}(t)=\frac{\left(P_{i, q+1}-P_{i, q}\right)}{h}=\frac{L\left(P_{i, q+1}-P_{i, q}\right)}{\tau}=\Psi_{i}^{(q)}, \quad t \in \mathcal{N}_{k, q} .
$$

Moreover, one has

$$
\begin{aligned}
2 \dot{x}^{T} & (t) \mathscr{P}_{i}(t) x(t) \\
& =2 \dot{x}^{T}(t) \mathscr{P}_{i}^{(q)}(\alpha) x(t) \\
& =x^{T}(t)\left[A_{i}^{T} \mathscr{P}_{i}^{(q)}(\alpha)+\mathscr{P}_{i}^{(q)}(\alpha) A_{i}\right] x(t), \quad t \in \mathcal{N}_{k, q} .
\end{aligned}
$$

By the linear interpolation relationship as (3), note that for $t \in \mathcal{N}_{k, q}$, we see

$$
\begin{aligned}
A_{i}^{T} \mathscr{P}_{i}^{(q)} & (\alpha)+\mathscr{P}_{i}^{(q)}(\alpha) A_{i}+\Psi_{i}^{(q)} \\
= & (1-\alpha)\left[A_{i}^{T} P_{i, q}+P_{i, q} A_{i}+\Psi_{i}^{(q)}\right] \\
& +\alpha\left[A_{i}^{T} P_{i, q+1}+P_{i, q+1} A_{i}+\Psi_{i}^{(q)}\right] .
\end{aligned}
$$

Thus, from (6) and (7), we get

$$
\begin{array}{r}
\dot{V}_{i}(t)=x^{T}(t)\left[A_{i}^{T} \mathscr{P}_{i}^{(q)}(\alpha)+\mathscr{P}_{i}^{(q)}(\alpha) A_{i}+\Psi_{i}^{(q)}\right] x(t)<0, \\
t \in \bigcup_{q=0, \ldots, L-1} \mathcal{N}_{k, q}=\left[t_{k}, t_{k}+\tau\right) .
\end{array}
$$

On the other hand, since $\mathscr{P}_{i}(t)=P_{i, L}$, when $t \in\left[t_{k}+\right.$ $\left.\tau, t_{k+1}\right)$, it yields $\dot{V}_{i}(t)=x^{T}(t)\left(A_{i}^{T} P_{i, L}+P_{i, L} A_{i}\right) x(t), t \in\left[t_{k}+\right.$ $\left.\tau, t_{k+1}\right)$. By (8), $\dot{V}_{i}(t)<0, t \in\left[t_{k}+\tau, t_{k+1}\right)$. Thus, we can conclude that

$$
\dot{V}_{i}(t)<0, \quad \forall t \in\left[t_{k}, t_{k+1}\right) .
$$

For overall switched system (1), we choose Lyapunov function described by

$$
V(t)=\sum_{i \in \mathscr{I}} \theta_{i}(t) V_{i}(t)=\sum_{i \in \mathscr{I}} x^{T}(t) \theta_{i}(t) \mathscr{P}_{i}(t) x(t),
$$

where $\theta_{i}(\cdot): \mathbb{R}^{+} \rightarrow\{0,1\}$ and $\sum_{i \in \mathcal{F}} \theta_{i}(t)=1$. Then, by the structure of discretized matrix function $\mathscr{P}_{i}(t), i \in \mathscr{I}$, and (9), one has

$$
V^{+}\left(t_{k}\right)-V^{-}\left(t_{k}\right) \leq 0, \quad \forall t_{k} \in \mathcal{S},
$$

where $V^{+}\left(t_{k}\right)=V_{j}\left(t_{k}\right)$ and $V^{-}\left(t_{k}\right)=V_{i}\left(t_{k}\right)$, if system switches from $i$ to $j$ at switching instant $t_{k}$. Combining (15) and (17), $V(t)$ is decreasing along with the time and converges to zero as $t \rightarrow \infty$. The GUAS of system (1) under switching signal with dwell time $\tau$ can be established.

Remark 7. Here it has to be noted that the number of discretized matrices $P_{i, q}, q=0,1, \ldots, L$ is $L+1$ which has to be prescribed in advance, and different choices of $L$ could lead to different analysis results. Roughly speaking, the larger $L$ is chosen; a denser division of dwell-time interval $\left[t_{k}, t_{k}+\tau\right)$ can be produced and, intuitively, a less conservative result can be obtained, which will be demonstrated by numerical example later. However, these less conservative results obtained by larger $L$ will directly lead to increase of computational complexity.

Remark 8 . The following property can hold if Theorem 6 holds for some $\tau^{*}$, then it holds for any $\tau \geq \tau^{*}$. Since Theorem 6 holds for some $\tau^{*}$, we can still choose interval $\left[t_{k}, t_{k}+\tau^{*}\right)$ to be divided into same segments $\mathcal{N}_{k, q}, q=$ $0,1, \ldots, L-1$; then, (6) and (7) can guarantee the $t \in$ $\bigcup_{q=0,1, \ldots, L-1} \mathcal{N}_{k, q}=\left[t_{k}, t_{k}+\tau^{*}\right)$. And (8) has $\dot{V}_{i}(t)<$ $0, \forall t \in\left[t_{k}+\tau^{*}, t_{k+1}\right)=\left[t_{k}+\tau^{*}, t_{k}+\tau\right) \cup\left[t_{k}+\right.$ $\left.\tau, t_{k+1}\right)$. Thus, $\dot{V}_{i}(t)<0, \forall t \in\left[t_{k}, t_{k+1}\right)$, can be also obtained to establish the stability for any $\tau \geq \tau^{*}$, and the minimal admissible dwell time guaranteeing system (1) GUAS can be estimated by

$$
\tau^{*}=\min _{\tau>0}\{\tau:(6)-(9) \text { hold }\} .
$$

When we choose $L=0$ indicating $\Psi_{i}^{(q)}=0$, along with (9), Theorem 6 is reduced to common Lyapunov function approach.

Since the discretized Lyapunov function contains the information of dwell time in its own structure, the estimation of dwell time is automatically executed by solving a set of LMIs without any additional nonconvex relations such as $\tau>\tau^{*}=\ln \mu / \lambda$ as in Lemma 3 , where $\mu$ and $\lambda$ have to be chosen manually. This feature makes our approach easily used with least conservativeness in the framework of discretized Lyapunov function approach. 
Example 9. A continuous-time switched linear system is given as $\dot{x}(t)=A_{i} x(t), i=1,2$, where

$$
\begin{aligned}
& A_{1}=\left[\begin{array}{ccc}
0.38 & 0.03 & -0.80 \\
0.96 & -0.92 & -1.66 \\
0.81 & -0.11 & -0.90
\end{array}\right], \\
& A_{2}=\left[\begin{array}{ccc}
-0.26 & 0.98 & 0.23 \\
-1.18 & -0.51 & 0.02 \\
-2.20 & 0.32 & -1.00
\end{array}\right] .
\end{aligned}
$$

Hereby, we are focusing on the important parameter $L$ concerned with division of dwell time interval in the context of continuous-time case and to show how $L$ affects the result obtained by Theorem 6 .

In Table 1, we see that the minimal admissible dwell time tends to smaller value as $L$ is increased, which implies, as what Remark 7 states, the larger $L$ concerned with a denser division of dwell time interval can lead to a less conservative result.

\section{Disturbance Attenuation Performance Analysis}

As what is discussed in Section 1, the disturbance attenuation property analysis in the sense of $\mathscr{L}_{2}$ gain performance related to dwell time has been viewed as an open problem. The general property relationship between the $\mathscr{L}_{2}$ induced gain and dwell time can be described as a function $\psi:[0, \infty) \rightarrow$ $[0, \infty)$ that maps each dwell time $\tau$ with $\mathscr{L}_{2}$ induced gain $\gamma^{*}$ of switched system (1), for the dwell-time switching signal $\sigma(t) \in \mathscr{D}_{\tau}$. For $\psi(\tau)$, at least the following two obvious observations are known from [27]:

(i) $\psi(\tau)$ is monotonically decreasing;

(ii) $\psi(\tau)$ is bounded below by $\tilde{\gamma}^{*}=\sup _{i \in \mathcal{F}}\left\{\gamma_{i}^{*}\right\}$, where $\gamma_{i}^{*}$ is the $\ell_{2}$ induced gain ( $\mathscr{L}_{2}$ induced gain) of $i$ th subsystem.

The first property is a trivial consequence of the fact that, given two dwell times $\tau_{2} \geq \tau_{1}$, we have that $D_{\tau_{2}} \subseteq D_{\tau_{1}}$. Then the second property results from the fact that, for every switching set $\mathscr{D}_{\tau}, \forall \tau>0$ contains all the constant switching signals $\sigma(t)=i, i \in \mathscr{I}$.

However, the above observations on function $\psi(\tau)$ are trivial for solving disturbance attenuation problem, where the quantitative relationship is required. In this section, we are going to present a method to compute $\psi(\tau)$ quantitatively, which solves disturbance attenuation problem.

Theorem 10. Consider the switched linear system (1). If there exist a set of matrices $P_{i, q}>0, q=0,1, \ldots, L, i \in \mathscr{I}$, such that

$$
\begin{aligned}
& {\left[\begin{array}{ccc}
\operatorname{He}\left\{P_{i, q} A_{i}\right\}+\Psi_{i}^{(q)} & P_{i, q} B_{i} & C_{i}^{T} \\
* & -\gamma^{2} I & D_{i}^{T} \\
* & * & -I
\end{array}\right]<0} \\
& q=0,1, \ldots, L-1, \forall i \in \mathscr{I} \\
& {\left[\begin{array}{ccc}
\mathrm{He}\left\{P_{i, q+1} A_{i}\right\}+\Psi_{i}^{(q)} & P_{i, q+1} B_{i} & C_{i}^{T} \\
* & -\gamma^{2} I & D_{i}^{T} \\
* & * & -I
\end{array}\right]<0,} \\
& q=0,1, \ldots, L-1, \forall i \in \mathcal{I}, \\
& {\left[\begin{array}{ccc}
A_{i}^{T} P_{i, L}+P_{i, L} A_{i} & P_{i, L} B_{i} & C_{i}^{T} \\
* & -\gamma^{2} I & D_{i}^{T} \\
* & * & -I
\end{array}\right]<0, \quad \forall i \in \mathscr{I},} \\
& P_{j, 0}-P_{i, L} \leq 0, \quad i \neq j, \forall i, j \in \mathscr{I},
\end{aligned}
$$

where $\Psi_{i}^{(q)}=L\left(P_{i, q+1}-P_{i, q}\right) / \tau$. Then switched system (1) is GUAS when $\omega(t)=0$ and has an $\mathscr{L}_{2}$ gain $\gamma$ with a dwell time $\tau$.

Proof. Let $J=\int_{t=0}^{\infty}\left[y^{T}(t) y(t)-\gamma^{2} \omega^{T}(t) \omega(t)\right] \mathrm{d} t$ and define $V(t)$ same as (16) on the basis of discretized Lyapunov function in continuous-time case (5). From (23), it is easy to see that $V^{+}\left(t_{k}\right)-V^{-}\left(t_{k}\right) \leq 0, \forall t_{k}$, where $V^{+}\left(t_{k}\right)$ and $V^{-}\left(t_{k}\right)$ are defined same as in (17). Then following result can be derived with assumption $x(0)=0$; the following derivation can be obtained:

$$
\begin{aligned}
J & =\sum_{k=0}^{\infty}\left(\int_{t=t_{k}}^{t_{k+1}} \Gamma(t) \mathrm{d} t+\left[V^{+}\left(t_{k}\right)-V^{-}\left(t_{k+1}\right)\right]\right) \\
& =\sum_{k=0}^{\infty}\left(\int_{t=t_{k}}^{t_{k+1}} \Gamma(t) \mathrm{d} t\right)+\sum_{k=1}^{\infty}\left[V^{+}\left(t_{k}\right)-V^{-}\left(t_{k}\right)\right] \\
& \leq \sum_{k=0}^{\infty}\left(\sum_{q=0}^{L-1}\left(\int_{t=t_{k}+\theta_{q}}^{t_{k}+\theta_{q+1}} \Gamma(t) \mathrm{d} t\right)+\int_{t=t_{k}+\tau}^{t_{k+1}} \Gamma(t) \mathrm{d} t\right),
\end{aligned}
$$

where $\Gamma(t)=y^{T}(t) y(t)-\gamma^{2} \omega^{T}(t) \omega(t)+\dot{V}(t)$. For $t \in \mathcal{N}_{k, q} \subset$ $\left[t_{k}, t_{k}+\tau\right)$, we see

$$
\begin{aligned}
\Gamma(t) & =\xi^{T}(t)\left[\begin{array}{cc}
\mathscr{P}_{i}(t)+A_{i}^{T} \mathscr{P}_{i}(t)+\mathscr{P}_{i}(t) A_{i}+C_{i}^{T} C_{i} & \mathscr{P}_{i}(t) B_{i}+C_{i}^{T} D_{i} \\
* & D_{i}^{T} D_{i}-\gamma^{2} I
\end{array}\right] \xi(t) \\
& =\xi^{T}(t)\left[(1-\alpha) \Gamma_{i, 1}^{(q)}+\alpha \Gamma_{i, 2}^{(q)}\right] \xi(t),
\end{aligned}
$$


TABle 1: Admissible dwell time with different $L$.

\begin{tabular}{ccccccccccc}
\hline$L$ & 1 & 2 & 3 & 4 & 5 & 6 & 7 & 8 & 9 & 10 \\
\hline$\tau^{*}$ & 3.396 & 2.599 & 2.342 & 2.219 & 2.140 & 2.084 & 2.038 & 1.997 & 1.958 & 1.927 \\
\hline
\end{tabular}

where $0 \leq \alpha \leq 1, \xi^{T}(t)=\left[x^{T}(t) \omega^{T}(t)\right], \Psi_{i}^{(q)}=L\left(P_{i, q+1}-\right.$ $\left.P_{i, q}\right) / \tau$, and

$$
\begin{gathered}
\Gamma_{i, 1}^{(q)}=\left[\begin{array}{cc}
\operatorname{He}\left\{A_{i}^{T} P_{i, q}\right\}+C_{i}^{T} C_{i}+\Psi_{i}^{(q)} & P_{i, q} B_{i}+C_{i}^{T} D_{i} \\
* & D_{i}^{T} D_{i}-\gamma^{2} I
\end{array}\right] \\
\Gamma_{i, 2}^{(q)}=\left[\begin{array}{cc}
\operatorname{He}\left\{P_{i, q+1} A_{i}\right\}+C_{i}^{T} C_{i}+\Psi_{i}^{(q)} & P_{i, q+1} B_{i}+C_{i}^{T} D_{i} \\
* & D_{i}^{T} D_{i}-\gamma^{2} I
\end{array}\right] .
\end{gathered}
$$

Then, by Schur complement formula, (20) and (21) guarantee $\Gamma_{i, 1}^{(q)}<0$ and $\Gamma_{i, 2}^{(q)}<0$ for $\forall q=0,1, \ldots, L$ and $\forall i \in \mathscr{I}$, respectively. Thus,

$$
\Gamma(t)<0, \quad \forall t \in \bigcup_{q=0,1, \ldots, L-1} \mathcal{N}_{k, q}=\left[t_{k}, t_{k}+\tau\right)
$$

And when $t \in\left[t_{k}+\tau, t_{k+1}\right),(22)$ also ensures

$$
\Gamma(t)<0, \quad \forall t \in\left[t_{k}+\tau, t_{k+1}\right) .
$$

Thus, combining (27) and (28) implies $J<0$. Therefore, the prescribed $\mathscr{L}_{2}$ gain performance $\|y(t)\|_{\mathscr{L}_{2}} \leq \gamma\|\omega(t)\|_{\mathscr{L}_{2}}$ is guaranteed, and GUAS with $\omega(t)=0$ is established by $\dot{V}(t)<$ $-y^{T}(t) y(t)<0, \forall t \in\left[t_{k}, t_{k+1}\right)$, and $V^{+}\left(t_{k}\right)-V^{-}\left(t_{k}\right) \leq 0, \forall t_{k}$ by $(23)$.

Remark 11. The nonincreasing and bounded properties with relationship between $\mathscr{L}_{2}$ induced gain and dwell time hold for continuous-time case through considering optimization problem

$$
\begin{array}{ll}
\min & \gamma^{2} \\
\text { s.t. } & (20)-(23) .
\end{array}
$$

Furthermore, similar to the stability analysis case, the $L$ related to the division of dwell time interval has to be prespecified, and, obviously, if a larger $L$ is chosen which implies a denser division, a more precise characterization can be attained.

Similar to the discussion in Remark 8 for stability, if $\tau^{*}$ is a dwell time such that the optimization (29) is feasible, so is any $\tau \geq \tau^{*}$. Furthermore, since $\mathscr{D}_{\tau} \subseteq \mathscr{D}_{\tau^{*}}$ for any $\tau \geq \tau^{*}$, we infer that the minimal value of $\gamma$ for solving (29) is nonincreasing as dwell time $\tau$ increases, and the minimal admissible dwell time guaranteeing optimization (29) feasible is

$$
\tau^{*}=\min _{\tau>0}\{\tau:(20) \text { holds }\}
$$

In addition, by the well-known results for a single system, the $\ell_{2}$ induced gain of $i$ th subsystem $\gamma_{i}^{*}$ can be simply figured out as $\min \gamma^{2}$ s.t. (22) for each subsystem. Compared with optimization (29), it is obvious that we have $\gamma^{*} \geq \gamma_{i}^{*}, \forall i \in \mathscr{I}$, which means the $\mathscr{L}_{2}$ gain performance is worsened by the occurrence of switching, and hence the $\mathscr{L}_{2}$ induced gain $\gamma^{*}$ is bounded below by $\tilde{\gamma}^{*}=\sup _{i \in \mathscr{I}}\left\{\gamma_{i}^{*}\right\}$.

Noting the sufficient conditions in Theorems 6 and 10, the LMIs are all affine in system matrices. As a result, many other existing standard techniques can be easily employed into Theorems 6 and 10; for example, when the system matrices contain uncertainties, the standard techniques such as [35] dealing additive uncertainties or [17] for polytopic uncertainties can be straightforwardly used in Theorems 6 and 10 . Thus, the discretized Lyapunov function approach not only obtains results for stability and disturbance attenuation issues, but also provides a general convex tool for dwell-time constrained switched systems.

\section{Application in $\mathscr{H}_{\infty}$ Control Synthesis}

Consider the control synthesis problem, the following switched linear system with control input is given as

$$
\begin{aligned}
& \dot{x}(t)=A_{\sigma(t)} x(t)+B_{\sigma(t)} u(t)+E_{\sigma(t)} \omega(t), \\
& y(t)=C_{\sigma(t)} x(t)+D_{\sigma(t)} \omega(t)+F_{\sigma(t)} \omega(t),
\end{aligned}
$$

where $u(t) \in \mathbb{R}^{l}$ is the control input, $x(t), \omega(t)$, and $y(t)$ are defined the same as in (1). As for switched system (31), the control synthesis problem basically involves controller and switching law design. By the previous results for dwell-time constrained switched system, such as Lemma 3, due to its nonconvex nature, most of the control synthesis procedures only have to be executed undesirably by two separate steps:

(i) design controllers for subsystems which are obtained at first step;

(ii) design the switching law; that is, determine the admissible dwell time, for the closed-loop system in the second step.

The two-step design method abruptly splits the controller and switching law design into two independently parts, which ought to be related to each other, and thus it often leads to conservative results for control problems under the dwell time constraint.

In this section, we are going to establish a one-step design methodology, by which the controller can be determined with respect to each specific dwell time, or in other words, the controller and dwell time can be determined simultaneously. We note that the information of dwell time is involved within the LMI-based convex sufficient conditions proposed in 
previous sections; this important feature could inspire us to use the discretized Lyapunov function techniques to build the one-step design framework.

Hereby, we are going to formulate the controller of discretized time-scheduled structure; then, the controller and switching law can be obtained by one unified step which explicitly facilitates the control synthesis process. Assuming the switching between system and the corresponding controller is synchronous, the mode-dependent time-scheduled state feedback controller which we are interested in is given in the form of

$$
u(t)=\mathscr{K}_{\sigma(t)}(t) x(t)
$$

where $\mathscr{K}_{i}(t), i \in \mathscr{I}$, are time-scheduled gains that have to be determined. After substituting controller (32) into system (31), the closed-loop system becomes

$$
\begin{aligned}
& \dot{x}(t)=\left[A_{\sigma(t)}+B_{\sigma(t)} \mathscr{K}_{\sigma(t)}(t)\right] x(t)+E_{\sigma(t)} \omega(t), \\
& y(t)=\left[C_{\sigma(t)}+D_{\sigma(t)} \mathscr{K}_{\sigma(t)}(t)\right] x(t)+F_{\sigma(t)} \omega(t) .
\end{aligned}
$$

As far as we know, due to the unsolved open problem on $\mathscr{L}_{2}$ gain, few results about relevant $\mathscr{H}_{\infty}$ control problem under dwell time constraint have been reported so far, though there exist numerous papers which are concerned with the weighted $\mathscr{H}_{\infty}$ performance based on Lemma 3. Some previous results $[26,36]$, claimed that the $\mathscr{H}_{\infty}$ control performance can be obtained by a straightforward extension from Lemma 3. Unfortunately, these results suffer from a mistake that the desired $\mathscr{H}_{\infty}$ performance should be replaced by weighted $\mathscr{H}_{\infty}$ performance; readers can refer to the recent comment paper [30]; however, the nonweighted $\mathscr{H}_{\infty}$ control problem performance related to dwell time still remains unsolved.

In this section, inspired by the discretized Lyapunov function approach presented in previous section, the sufficient conditions for the existence of $\mathscr{H}_{\infty}$ controller of switched system (31).

Motivated by the discretized Lyapunov function technique in continuous-time case, we define the following two time-scheduled matrices $\mathcal{S}_{i}(t)$ and $\mathscr{X}_{i}(t), i \in \mathscr{I}$, which is discretized as follows:

$$
\begin{aligned}
& \mathcal{S}_{i}(t) \\
& =\left\{\begin{array}{ll}
\mathcal{S}_{i}^{(q)}(\alpha) & q=0,1, \ldots, L-1, t \in \mathcal{N}_{k, q} \\
S_{i, L} & t \in\left[t_{k}+\tau, t_{k+1}\right),
\end{array} \quad i \in \mathscr{I},\right. \\
& \mathscr{X}_{i}(t) \\
& = \begin{cases}X_{i}^{(q)}(\alpha) & q=0,1, \ldots, L-1, t \in \mathcal{N}_{k, q} \\
X_{i, L} & t \in\left[t_{k}+\tau, t_{k+1}\right),\end{cases}
\end{aligned}
$$

where $S_{i, q}>0, \mathcal{S}_{i}^{(q)}(\alpha)=\mathcal{S}_{i}\left(t_{k}+\theta_{q}+\alpha h\right)=(1-\alpha) S_{i, q}+\alpha S_{i, q+1}$, $\mathscr{X}_{i}^{(q)}(\alpha)=\mathscr{X}_{i}\left(t_{k}+\theta_{q}+\alpha h\right)=(1-\alpha) X_{i, q}+\alpha X_{i, q+1}$, and $\alpha$, $\theta_{q}, h, \mathcal{N}_{k, q}$ are defined the same as in (3). Then, with the aid of above discretized matrices, the following theorem can be obtained.

Theorem 12. Consider switched system (1) with dwell time $\tau$. If there exist a set of matrices $S_{i, q}, X_{i, q}, q=0,1, \ldots, L, \forall i \in \mathscr{I}$, such that

$$
\begin{aligned}
& {\left[\begin{array}{ccc}
\Xi_{i, 1}^{(q)} & E_{i} & S_{i, q} C_{i}^{T}+X_{i, q}^{T} D_{i}^{T} \\
* & -\gamma^{2} I & F_{i}^{T} \\
* & * & -I
\end{array}\right]<0,} \\
& q=0,1, \ldots, L-1, \forall i \in \mathscr{I}, \\
& {\left[\begin{array}{ccc}
\Xi_{i, 2}^{(q)} & E_{i} & S_{i, q+1} C_{i}^{T}+X_{i, q+1}^{T} D_{i}^{T} \\
* & -\gamma^{2} I & F_{i}^{T} \\
* & * & -I
\end{array}\right]<0,} \\
& q=0,1, \ldots, L-1, \forall i \in \mathscr{I}, \\
& {\left[\begin{array}{ccc}
\Xi_{i, 3}^{(L)} & E_{i} & S_{i . L} C_{i}^{T}+X_{i . L}^{T} D_{i}^{T} \\
* & -\gamma^{2} I & F_{i}^{T} \\
* & * & -I
\end{array}\right]<0,} \\
& \forall i \in \mathscr{I} \text {, } \\
& {\left[\begin{array}{cc}
-S_{j, L} & S_{j, L} \\
* & -S_{i, 0}
\end{array}\right] \leq 0, \quad i \neq j, \quad \forall i, j \in \mathscr{I},}
\end{aligned}
$$

where $\Xi_{i, 1}^{(q)}=\operatorname{He}\left\{A_{i} S_{i, q}+B_{i} X_{i, q}\right\}-\Psi_{i}^{(q)}, \Xi_{i, 2}^{(q)}=\operatorname{He}\left\{A_{i} S_{i, q+1}+\right.$ $\left.B_{i} X_{i, q+1}\right\}-\Psi_{i}^{(q)}, \Xi_{i, 3}^{(L)}=\operatorname{He}\left\{A_{i} S_{i, L}+B_{i} X_{i, L}\right\}$, and $\Psi_{i}^{(q)}=$ $L\left(S_{i, q+1}-S_{i, q}\right) / \tau$. Then the closed-loop system (33) with controller (32) is GUAS when $\omega(t)=0$ and has an $\mathscr{L}_{2}$ gain $\gamma$, and the controller gain is given as

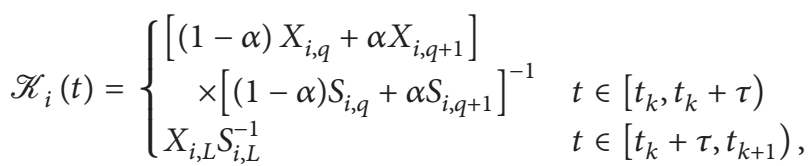

where $\alpha=L\left(t-t_{k}\right) / \tau-q$.

Proof. From the guidelines in Theorem 10, the key point is to prove the following inequality:

$$
\left[\begin{array}{cc}
\dot{\mathscr{P}}_{i}(t)+\operatorname{He}\left\{\mathscr{P}_{i}(t) \mathscr{A}_{i}(t)\right\}+\mathscr{C}_{i}^{T}(t) \mathscr{C}_{i}(t) & \mathscr{P}_{i}(t) E_{i}+\mathscr{C}_{i}^{T}(t) D_{i} \\
* & F_{i}^{T} F_{i}-\gamma^{2} I
\end{array}\right]<0,
$$


where $\mathscr{A}_{i}(t)=A_{i}+B_{i} \mathscr{K}_{i}(t)$ and $\mathscr{C}_{i}(t)=C_{i}+D_{i} \mathscr{K}_{i}(t)$. We can choose $\mathscr{P}_{i}(t)=\mathcal{S}_{i}^{-1}(t)$, where $\mathcal{S}_{i}(t), i \in \mathscr{I}$, is defined by (34).
And using the fact $\dot{\mathscr{P}}_{i}(t)=\dot{\mathcal{S}}_{i}^{-1}(t)=-\mathcal{S}_{i}^{-1}(t) \dot{\mathcal{S}}_{i}(t) \mathcal{S}_{i}^{-1}(t),(41)$ is equivalent to

$$
\left[\begin{array}{cccc}
\operatorname{He}\left\{\mathcal{S}_{i}^{-1}(t) \mathscr{A}_{i}(t)\right\} & -\mathcal{S}_{i}^{-1}(t) \dot{\mathcal{S}}_{i}(t) \mathcal{S}_{i}^{-1}(t) & \mathcal{S}_{i}^{-1}(t) B_{i} & C_{i}^{T} \\
* & -\gamma^{2} I & D_{i}^{T} \\
* & * & -I
\end{array}\right]<0 .
$$

Multiplying both sides of the previous LMI by $\operatorname{diag}\left\{\mathcal{S}_{i}(t), I, I\right\}$, and due to the controller structure (40), we have $\mathscr{X}_{i}(t)=\mathscr{K}_{i}(t) \mathcal{S}_{i}(t)$, where $\mathscr{X}_{i}(t)$ is given as (35); substituting it into (42), it becomes

$$
\left[\begin{array}{ccc}
\operatorname{He}\left\{\mathscr{A}_{i} \mathcal{S}_{i}(t)+B_{i} \mathscr{X}_{i}(t)\right\}-\dot{\mathcal{S}}_{i}(t) & E_{i} & \mathcal{S}_{i}(t) C_{i}^{T}+\mathscr{X}_{i}^{T}(t) D_{i}^{T} \\
* & -\gamma^{2} I & F_{i}^{T} \\
* & * & -I
\end{array}\right]<0 .
$$

Moreover, by the similar guidelines in Theorem 6, we observe that $\dot{\delta}_{i}(t)=L\left(S_{i, q+1}-S_{i, q}\right) / \tau=\Psi_{i}^{(q)}$. Thus, due to the structure of discretized $\mathcal{S}_{i}(t)$ and $\mathscr{X}_{i}(t)$, it is guaranteed that (43) can be established by (36)-(38).

Afterwards, by $S_{i, \tau}^{-1}=P_{i, \tau}$ and $S_{j, 0}^{-1}=P_{j, 0}$ and according to Schur complement formula, it is noted that

$$
\begin{aligned}
(39) & \Longleftrightarrow S_{i, L} S_{j, 0}^{-1} S_{i, L}-S_{i, L}<0 \\
& \Longleftrightarrow S_{i, L}\left(S_{j, 0}^{-1}-S_{i, L}^{-1}\right) S_{i, L}<0 \\
& \Longleftrightarrow S_{j, 0}^{-1}-S_{i, L}^{-1}<0 \\
& \Longleftrightarrow P_{j, 0}-P_{i, L}<0 .
\end{aligned}
$$

Then, together with $S_{i, q}>0 \Leftrightarrow P_{i, q}>0, q=0,1, \ldots, L$, $i \in \mathscr{I}$, we can conclude that the closed-loop system (33) is GUAS and has an $\mathscr{L}_{2}$ gain $\gamma$ with the controller gain (40).

Remark 13. The philosophy of one-step method is also applicable in continuous-time case with Theorem 12. Once the parameter $L$ is chosen, the controller gains are determined with respect to a specific dwell time by one step via solving LMIs (36)-(39). And the controller with optimal $\mathscr{H}_{\infty}$ control performance can be computed by

$$
\begin{array}{ll}
\min & \gamma^{2} \\
\text { s.t. } & (36)-(39)
\end{array}
$$

with respect to a specific dwell time $\tau$ and prescribed $L$. Similar to the discussion for disturbance attenuation analysis, a larger $L$ will yield a less conservative design result.

Example 14. The switched system considered is composed of two subsystems. The system matrices are given as follows: $A_{i}$, $i=1,2$, are same as the example in Section 3 , and $B_{i}, C_{i}, D_{i}$, $E_{i}$, and $F_{i}, i=1,2$ are given as

$$
\begin{array}{ll}
B_{1}=\left[\begin{array}{c}
1.31 \\
0.52 \\
-1.10
\end{array}\right], & C_{1}^{T}=\left[\begin{array}{c}
-1.42 \\
0.26 \\
-0.36
\end{array}\right], \quad D_{1}=0.54, \\
E_{1}=\left[\begin{array}{c}
-1.1 \\
2.04 \\
0.01
\end{array}\right], & F_{1}=1.08 \\
B_{2}=\left[\begin{array}{c}
0.81 \\
-1.22 \\
0.23
\end{array}\right], & C_{2}^{T}=\left[\begin{array}{c}
-0.98 \\
1.36 \\
0.86
\end{array}\right], \quad D_{2}=0.32, \\
E_{2}=\left[\begin{array}{c}
0.75 \\
0.54 \\
-0.69
\end{array}\right], & F_{2}=0.63 .
\end{array}
$$

The $\mathscr{H}_{\infty}$ control problem will be considered in the following example involved. The computation on optimal $\mathscr{H}_{\infty}$ control performance versus dwell time with difference $L$ is shown in Figure 1.

The relationship between dwell time and $\mathscr{H}_{\infty}$ induced gain is characterized in Figure 1. From the numerical results in Figure 1, we see following facts:

(i) the same monotonically decreasing property holds with any choices of $L$;

(ii) better results such as the smaller admissible dwell time and smaller disturbance attenuation level can be achieved by a larger $L$, which implies a denser division of dwell time interval leads to a less conservative analysis result. 


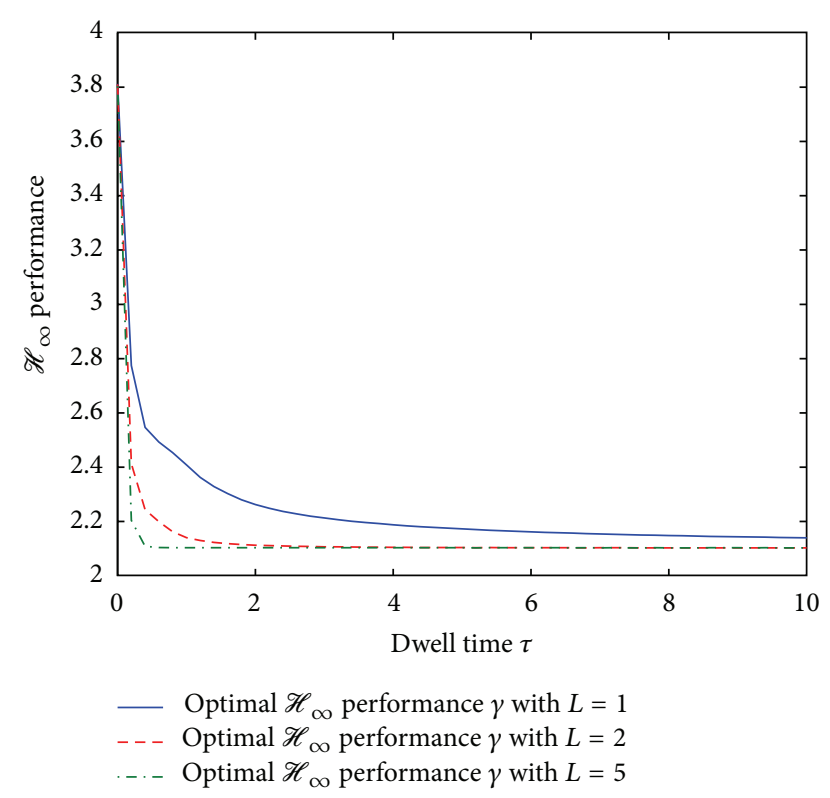

FIgURE 1: Computation on optimal $\mathscr{H}_{\infty}$ control performance versus dwell time with different $L$.

Taking a specific dwell time $\tau=4$ with $L=2$, the time-scheduled gains $\mathscr{K}_{i}(t), i=1,2$ with optimal $\mathscr{H}_{\infty}$ performance $\gamma^{*}=2.1129$ are determined by

$$
\mathscr{K}_{i}(t)= \begin{cases}{\left[(1-\alpha) X_{i, 0}+\alpha X_{i, 1}\right]} & \\ \quad \times\left[(1-\alpha) S_{i, 0}+\alpha S_{i, 1}\right]^{-1} & t \in\left[t_{k}, t_{k}+2\right) \\ {\left[(1-\alpha) X_{i, 1}+\alpha X_{i, 2}\right]} & \\ \quad \times\left[(1-\alpha) S_{i, 1}+\alpha S_{i, 2}\right]^{-1} & t \in\left[t_{k}+2, t_{k}+4\right) \\ X_{i, 2} S_{i, 2}^{-1} & t \in\left[t_{k}+4, t_{k+1}\right),\end{cases}
$$

where $\alpha=\left\{\begin{array}{cc}\left(t-t_{k}\right) / 2 & t \in\left[t_{k}, t_{k}+2\right) \\ \left(t-t_{k}\right) / 2-1 & t \in\left[t_{k}+2, t_{k}+4\right)\end{array}\right.$, and $S_{i, q}$ and $X_{i, q}, q=0,1,2$ and $i=1,2$, can be easily obtained by solving optimization problem $\min \gamma^{2}$ s.t.(36)-(39), which are omitted here.

\section{Conclusions}

The stability and disturbance attenuation properties for switched system with dwell time constraint are investigated in this paper. By introducing the discretized Lyapunov function technique, sufficient convex conditions ensure that the switched system GUAS are derived for continuous-time case. Then, the idea of discretized Lyapunov function is further extended to disturbance attenuation performance analysis in the sense of $\mathscr{L}_{2}$ gain, which has been viewed as an open problem not completely solved so far. Due to the convex feature of analysis results on the basis of discretized Lyapunov function, the $\mathscr{H}_{\infty}$ control synthesis problem is taken into consideration, and a one-step design method is proposed, which has obvious advantages over conventional two-step design method. By one-step design method, the controllers for subsystems and the switching law can be determined simultaneously, which facilitates the design process significantly. As what has been discussed in this paper, the discretized Lyapunov function technique can not only derive results for stability and disturbance attenuation issues, but also provide a general convex tool for dwell-time constrained switched systems, which can be used to studied other issues for switched systems in our future work.

\section{Conflict of Interests}

The authors declare that there is no conflict of interests regarding the publication of this paper.

\section{Acknowledgments}

This work is supported by Sichuan Provincial Education Department (no. 14ZB0266) and the School of Education Reform Project mnu-JY1408.

\section{References}

[1] D. Liberzon, Switching in Cystems and Control, Birkhäuser, Boston, Mass, USA, 2003.

[2] Z. Sun and S. Ge, Switched Linear Systems-Control and Design, Springer, London, UK, 2005.

[3] R. A. Decarlo, M. S. Branicky, S. Pettersson, and B. Lennartson, "Perspectives and results on the stability and stabilizability of hybrid systems," Proceedings of the IEEE, vol. 88, no. 7, pp. 1069$1082,2000$.

[4] H. Lin and P. J. Antsaklis, "Stability and stabilizability of switched linear systems: a survey of recent results," IEEE Transactions on Automatic Control, vol. 54, no. 2, pp. 308-322, 2009.

[5] A. Balluchi, M. D. Benedetto, C. Pinello, C. Ross, and A. Sangiovanni-Vincentelli, "Cut-off in engine control: a hybrid system approach," in Proceedings of the 36th IEEE Conference on Decision and Control, pp. 4720-4725, San Diego, Calif, USA, December 1997.

[6] B. E. Bishop and M. W. Spong, "Control of redundant manipulators using logic-based switching," in Proceedings of the 37th IEEE Conference on Decision and Control (CDC '98), pp. 14881493, Tampa, Fla, USA, December 1998.

[7] W. Zhang, M. S. Branicky, and S. M. Phillips, "Stability of networked control systems," IEEE Control Systems Magazine, vol. 21, no. 1, pp. 84-99, 2001.

[8] I. Kolmanovsky and S. Jing, "A multi-mode switching-based command tracking in network controlled systems with pointwise-in-time constraints and disturbance inputs," in Proceedings of the 6th World Congress on Intelligent Control and Automation (WCICA '06), pp. 199-204, Dalian, China, June 2006.

[9] K. S. Narendra, O. A. Driollet, M. Feiler, and K. George, "Adaptive control using multiple models, switching and tuning," International Journal of Adaptive Control and Signal Processing, vol. 17, no. 2, pp. 87-102, 2003.

[10] B. Castillo-Toledo, S. Di Gennaro, A. G. Loukianov, and J. Rivera, "Hybrid control of induction motors via sampled closed representations," IEEE Transactions on Industrial Electronics, vol. 55, no. 10, pp. 3758-3771, 2008. 
[11] C. Sreekumar and V. Agarwal, "A hybrid control algorithm for voltage regulation in DC-DC boost converter," IEEE Transactions on Industrial Electronics, vol. 55, no. 6, pp. 2530-2538, 2008.

[12] R. Shorten, F. Wirth, O. Mason, K. Wulff, and C. King, "Stability criteria for switched and hybrid systems," SIAM Review, vol. 49, no. 4, pp. 545-592, 2007.

[13] M. S. Branicky, "Multiple Lyapunov functions and other analysis tools for switched and hybrid systems," IEEE Transactions on Automatic Control, vol. 43, no. 4, pp. 475-482, 1998.

[14] K. S. Narendra and J. A. Balakrishnan, "A common Lyapunov function for stable LTI systems with commuting A-matrices," IEEE Transactions on Automatic Control, vol. 39, no. 12, pp. 2469-2471, 1994.

[15] J. Daafouz, P. Riedinger, and C. Iung, "Stability analysis and control synthesis for switched systems: a switched Lyapunov function approach," IEEE Transactions on Automatic Control, vol. 47, no. 11, pp. 1883-1887, 2002.

[16] W. Xiang and J. Xiao, "Stabilization of switched continuoustime systems with all modes unstable via dwell time switching," Automatica, vol. 50, no. 3, pp. 940-945, 2014.

[17] L. I. Allerhand and U. Shaked, "Robust stability and stabilization of linear switched systems with dwell time," IEEE Transactions on Automatic Control, vol. 56, no. 2, pp. 381-386, 2011.

[18] J. C. Geromel and P. Colaneri, "Stability and stabilization of discrete time switched systems," International Journal of Control, vol. 79, no. 7, pp. 719-728, 2006.

[19] K. Hirata and J. P. Hespanha, " $\mathscr{L} 2$-induced gain analysis for a class of switched systems," in Proceeding of thr 48th IEEE Conference on Decision and Control held jointly with the 28th Chinese Control Conference (CDC/CCC '09), pp. 2138-2143, Shanghai, China, December 2009.

[20] K. Hirata and J. Hespanha, " $\mathscr{L}_{2}$-induced gain analysis of switched linear systems via finitely parametrized storage functions," in Proceedings of the 29th American Control Conference (ACC '10), pp. 4064-4069, Baltimore, Md, USA, June 2010.

[21] W. Xiang and J. Xiao, "Stability analysis and control synthesis of switched impulsive systems," International Journal of Robust and Nonlinear Control, vol. 22, no. 13, pp. 1440-1459, 2012.

[22] W. Xiang, J. Xiao, and M. N. Iqbal, "Asymptotic stability, $\ell_{2}$ gain, boundness analysis, and control synthesis for switched systems: a switching frequency approach," International Journal of Adaptive Control and Signal Processing, vol. 26, no. 4, pp. 350373, 2012.

[23] M. Margaliot and J. P. Hespanha, "Root-mean-square gains of switched linear systems: a variational approach," Automatica, vol. 44, no. 9, pp. 2398-2402, 2008.

[24] A. S. Morse, "Supervisory control of families of linear set-point controllers. I. Exact matching," IEEE Transactions on Automatic Control, vol. 41, no. 10, pp. 1413-1431, 1996.

[25] J. P. Hespanha and A. S. Morse, "Stability of switched systems with average dwell-time," in Proceedings of the 38th IEEE Conference on Decision and Control (CDC '99), pp. 2655-2660, Phoenix, Ariz, USA, December 1999.

[26] L. Zhang and P. Shi, "Stability, $l_{2}$-gain and asynchronous $H_{\infty}$ control of discrete-time switched systems with average dwell time," IEEE Transactions on Automatic Control, vol. 54, no. 9, pp. 2192-2199, 2009.

[27] J. Hespanha, " $L_{2}$-induced gains of switched linear systems," in Unsolved Problems in Mathematical Systems and Control Theory, V. D. Blondel and A. Megretski, Eds., pp. 131-133, Princeton University Press, Princeton, NJ, USA, 2003.
[28] G. Zhai, B. Hu, K. Yasuda, and A. N. Michel, "Disturbance attenuation properties of time-controlled switched systems," Journal of the Franklin Institute, vol. 338, no. 7, pp. 765-779, 2001.

[29] G. Zhai, B. Hu, K. Yasuda, and A. N. Michel, "Qualitative analysis of discrete-time switched systems," in Proceedings of the American Control Conference, pp. 1880-1885, May 2002.

[30] W. Xiang and J. Xiao, "Discussion on stability, $1_{2}$-gain and asynchronous $H_{\infty}$ control of discrete-time switched systems with average dwell time," IEEE Transactions on Automatic Control, vol. 57, no. 12, pp. 3259-3261, 2012.

[31] C. Cai, "Dwell-time approach to input-output stability properties for a class of discrete-time dynamical systems," Systems \& Control Letters, vol. 60, no. 6, pp. 383-389, 2011.

[32] J. C. Geromel and P. Colaneri, " $\mathscr{H}_{\infty}$ and dwell time specifications of continuous-time switched linear systems," IEEE Transactions on Automatic Control, vol. 55, no. 1, pp. 207-212, 2010.

[33] P. Colaneri, P. Bolzern, and J. C. Geromel, "Root mean square gain of discrete-time switched linear systems under dwell time constraints," Automatica, vol. 47, no. 8, pp. 1677-1684, 2011.

[34] K. Gu, V. L. Kharitonov, and J. Chen, Stability of Time-Delay Systems, Springer, Berlin, Germany, 2003.

[35] W. Xiang, J. Xiao, and M. N. Iqbal, "Robust observer design for nonlinear uncertain switched systems under asynchronous switching," Nonlinear Analysis: Hybrid Systems, vol. 6, no. 1, pp. 754-773, 2012.

[36] B. Lu and F. Wu, "Switching LPV control designs using multiple parameter-dependent Lyapunov functions," Automatica, vol. 40, no. 11, pp. 1973-1980, 2004. 


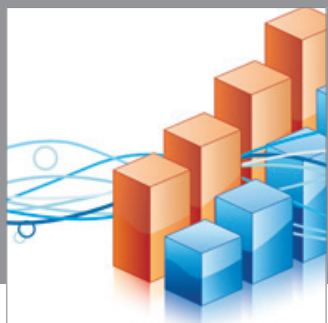

Advances in

Operations Research

mansans

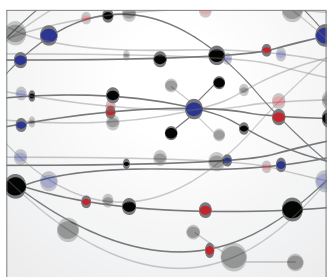

The Scientific World Journal
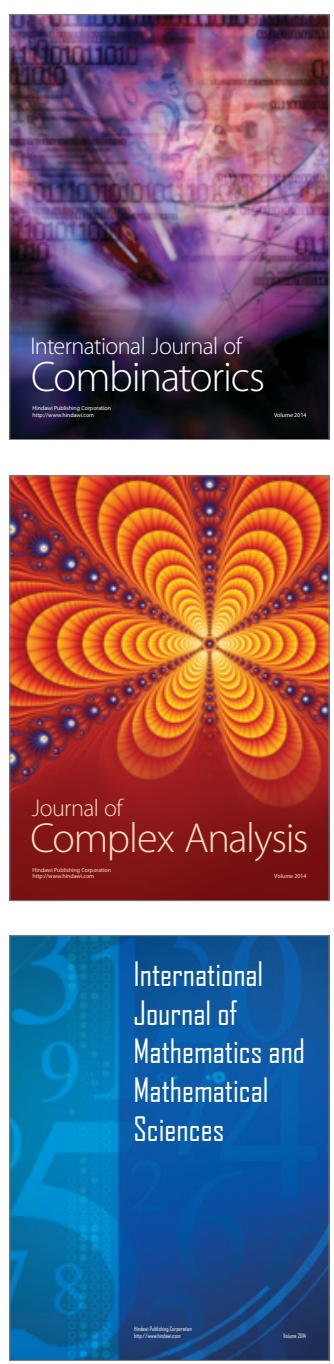
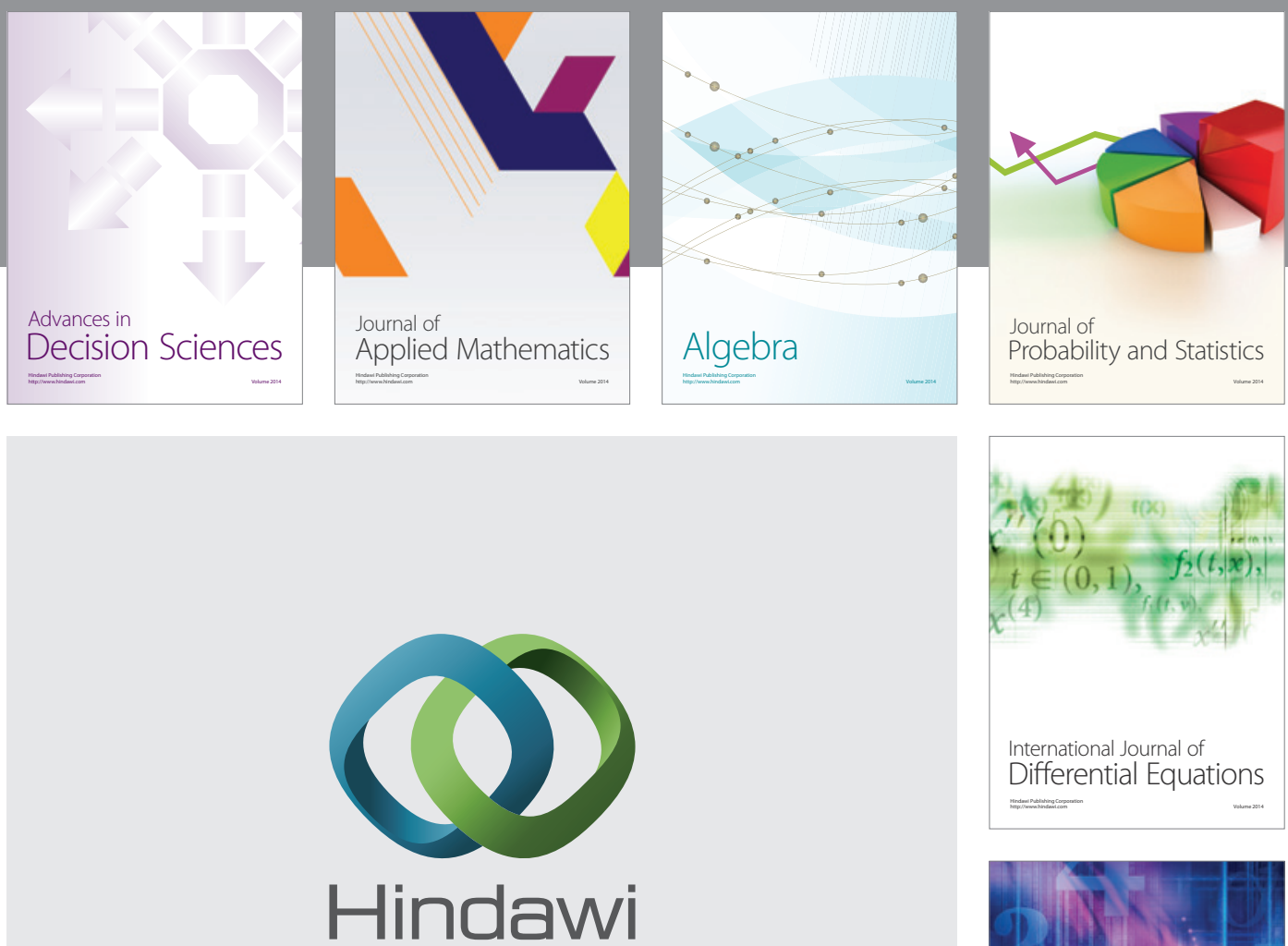

Submit your manuscripts at http://www.hindawi.com
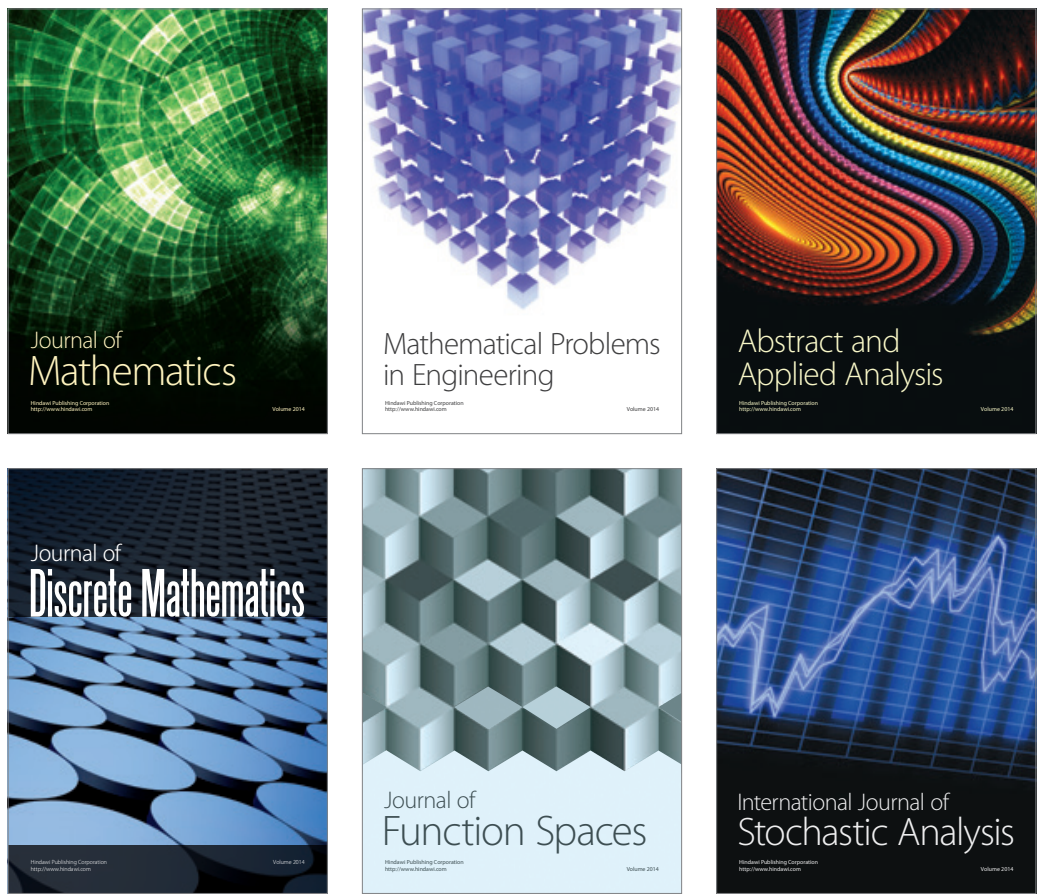

Journal of

Function Spaces

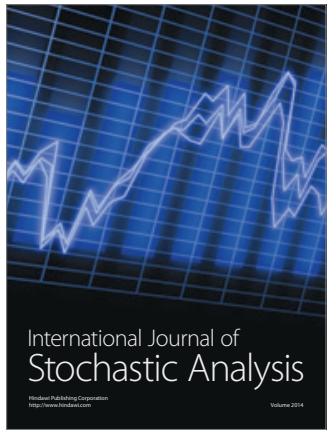

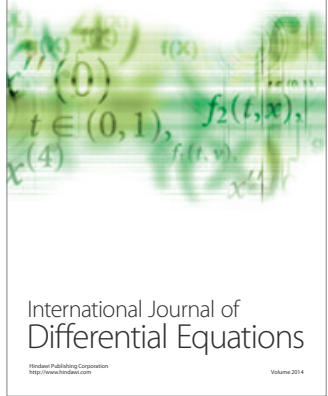
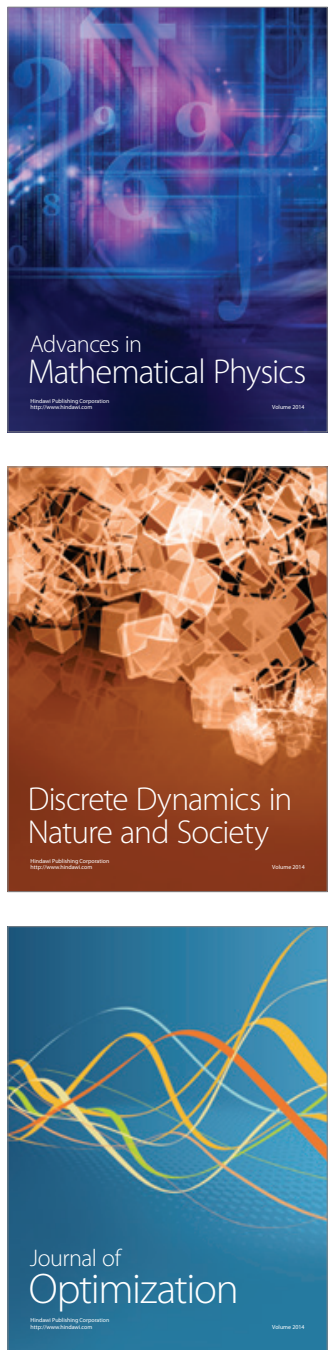